\title{
Statistics on the multi-colored permutation groups
}

\author{
Eli Bagno
}

The Jerusalem College of Technology, Jerusalem, Israel

bagnoe@jct.ac.il

Ayelet Butman

Department of Computer Science, Faculty of Sciences

Holon Institute of Technology, PO Box 305, 58102 Holon, Israel

ayeletb@it.ac.il

\section{David Garber}

Department of Applied Mathematics, Faculty of Sciences

Holon Institute of Technology, PO Box 305, 58102 Holon, Israel

garber@hit.ac.il

Submitted: Jan 14, 2007; Accepted: Feb 8, 2007; Published: Mar 5, 2007

Mathematics Subject Classification: 05E15; 05E99

\begin{abstract}
We define an excedance number for the multi-colored permutation group i.e. the wreath product $\left(\mathbb{Z}_{r_{1}} \times \cdots \times \mathbb{Z}_{r_{k}}\right)<S_{n}$ and calculate its multi-distribution with some natural parameters.

We also compute the multi-distribution of the parameters $\operatorname{exc}(\pi)$ and $\operatorname{fix}(\pi)$ over the sets of involutions in the multi-colored permutation group. Using this, we count the number of involutions in this group having a fixed number of excedances and absolute fixed points.
\end{abstract}

\section{Introduction}

Let $r_{1}, \ldots, r_{k}$ and $n$ be positive integers. The multi-colored permutation group $G_{r_{1}, \ldots, r_{k} ; n}$ is the wreath product:

$$
\left(\mathbb{Z}_{r_{1}} \times \mathbb{Z}_{r_{2}} \times \cdots \times \mathbb{Z}_{r_{k}}\right) \curlywedge S_{n}
$$

The symmetric group $S_{n}$ is a special case for $r_{i}=1,1 \leq i \leq k$. In $S_{n}$ one can define the following well-known parameters: Given $\sigma \in S_{n}, i \in[n]$ is an excedance of $\sigma$ if $\sigma(i)>i$. The number of excedances is denoted by $\operatorname{exc}(\sigma)$. Two other natural parameters on $S_{n}$ are 
the number of fixed points and the number of cycles of $\sigma$, denoted by fix $(\sigma)$ and $\operatorname{cyc}(\sigma)$ respectively.

Consider the following generating function over $S_{n}$ :

$$
P_{n}(q, t, s)=\sum_{\sigma \in S_{n}} q^{\operatorname{exc}(\sigma)} t^{\mathrm{fix}(\sigma)} s^{\operatorname{cyc}(\sigma)} .
$$

$P_{n}(q, 1,1)$ is the classical Eulerian polynomial, while $P_{n}(q, 0,1)$ is the counter part for the derangements, i.e. the permutations without fixed points, see [4].

In the case $s=-1$, the two polynomials $P_{n}(q, 1,-1)$ and $P_{n}(q, 0,-1)$ have simple closed formulas:

$$
\begin{gathered}
P_{n}(q, 1,-1)=-(q-1)^{n-1}, \\
P_{n}(q, 0,-1)=-q[n-1]_{q},
\end{gathered}
$$

where $[n]_{q}=\frac{q^{n}-1}{q-1}$.

Recently, Ksavrelof and Zeng [3] proved some new recursive formulas which induce the above equations. In [1], the corresponding excedance number for the colored permutation groups $G_{r, n}=\mathbb{Z}_{r} \prec S_{n}$ was defined. It was proved there that:

$$
\begin{gathered}
P_{G_{r, n}}(q, 1,-1)=\left(q^{r}-1\right) P_{G_{r, n-1}}(q, 1,-1), \\
P_{G_{r, n}}(q, 0,-1)=[r]_{q}\left(P_{G_{r, n-1}}(q, 0,-1)-q^{n-1}[r]_{q}^{n-1}\right),
\end{gathered}
$$

and hence,

$$
\begin{gathered}
P_{G_{r, n}}(q, 1,-1)=-\frac{\left(q^{r}-1\right)^{n}}{q-1}, \\
P_{G_{r, n}}(q, 0,-1)=-q[r]_{q}^{n}[n-1]_{q} .
\end{gathered}
$$

In this paper we generalize our parameters and formulas to the case of the multi-colored permutation groups. Explicitly, denote $r=r_{1} \cdots r_{k}$. We get the following theorems:

\section{Theorem 1.1.}

$$
P_{G_{r_{1}, \ldots, r_{k} ; n}}(q, 1,-1)=\left(q^{r}-1\right) P_{G_{r_{1}, \ldots, r_{k} ; n-1}}(q, 1,-1) .
$$

Hence,

$$
P_{G_{r_{1}, \ldots, r_{k} ; n}}(q, 1,-1)=(-1-K(q))\left(q^{r}-1\right)^{n-1},
$$

where

$$
K(q)=K\left(q ; r_{1}, \ldots, r_{k}\right)=\sum_{m=1}^{k} r_{m+1} \cdots r_{k} \sum_{t=1}^{r_{m}-1} q^{t \frac{r}{r_{m}}}
$$

For the derangements, we have: 
Theorem 1.2.

$$
P_{G_{r_{1}, \ldots, r_{k} ; n}}(q, 0,-1)=(1+K(q))\left(P_{G_{r_{1}, \ldots, r_{k} ; n-1}}(q, 0,-1)-\left(q^{r}+K(q)\right)^{n-1}\right) .
$$

Hence, we have:

$$
P_{G_{r_{1}, \ldots, r_{k} ; n}}(q, 0,-1)=\left(q^{r}+K(q)\right)(1+K(q)) \cdot\left((1+K(q))^{n-2}-\sum_{k=1}^{n-2}\left(q^{r}+K(q)\right)^{k}(1+K(q))^{n-2-k}\right)
$$

for all $n \geq 2$.

An element $\sigma$ in $G_{r_{1}, \ldots, r_{k} ; n}$ is called an involution if $\sigma^{2}=1$. The set of involutions in $G_{r_{1}, \ldots, r_{k} ; n}$ will be denoted by $I_{r_{1}, \ldots, r_{k} ; n}$.

In [2], the multi-distribution of the parameters exc, fix and csum on the set of involutions in the complex reflection groups was considered. We cite the following result from there. (The relevant definitions will be given in Section 6).

Theorem 1.3. (See Corollary 5.2 in [2])

The polynomial $\sum_{\pi \in G_{r, n}} u^{\mathrm{fix}(\pi)} v^{\operatorname{exc}_{\mathrm{A}}(\pi)} w^{\operatorname{csum}(\pi)}$ is given by

$$
\sum_{j=n / 2}^{n}(n-j) !\left(\begin{array}{c}
n \\
n-j, n-j, 2 j-n
\end{array}\right) \frac{u^{2 j-n}\left(v+(r-1) w^{r}\right)^{n-j}}{2^{n-j}} \mu_{r}^{2 j-n} .
$$

where $\mu_{r}=1$ if $r$ is odd, and $\mu_{r}=1+w^{\frac{r}{2}}$ otherwise.

Here, we generalize this result to $G_{r_{1}, \ldots, r_{k} ; n}$. We prove:

Theorem 1.4. The polynomial $\sum_{\pi \in G_{r_{1}, \ldots, r_{k} ; n}} u^{\mathrm{fix}(\pi)} v^{\operatorname{exc}_{\mathrm{A}}(\pi)} w^{\operatorname{csum}(\pi)}$ is given by

$$
\sum_{j=n / 2}^{n}(n-j) !\left(\begin{array}{c}
n \\
n-j, n-j, 2 j-n
\end{array}\right) \frac{u^{2 j-n}\left(v+(r-1) w^{r}\right)^{n-j}}{2^{n-j}} \mu^{2 j-n} .
$$

where $\mu=1$ if $r$ is odd, and $\mu=1+2^{\epsilon} w^{\frac{r}{2}}$ otherwise (where $\epsilon=\#\left\{r_{i} \mid 1 \leq i \leq k, r_{i} \equiv 0\right.$ $(\bmod 2)\})$. Hence, we have that the number of involutions $\pi \in G_{r_{1}, \ldots, r_{k} ; n}$ with $\operatorname{exc}(\pi)=m$ is:

$$
\left\{\begin{array}{clrl}
y !\left(\begin{array}{c}
n \\
y, y, n-2 y
\end{array}\right)\left(\frac{r}{2}\right)^{y} & r \equiv 1 & (\bmod 2) \\
\sum_{j=\frac{n}{2}}^{n}(n-j) !\left(\begin{array}{c}
n \\
n-j, n-j, j-y, y-n+j
\end{array}\right)\left(\frac{r}{2}\right)^{n-j} 2^{\epsilon(y-n+j)} & r \equiv 0 & (\bmod 2)
\end{array}\right.
$$

where $y=\frac{m}{r}$.

Note that every Abelian group $G$ can be presented as a direct product of cyclic groups, and thus this work generalizes the well-known excedance number to the wreath product of $S_{n}$ by any Abelian group. Nevertheless, this parameter depends on the order of the cyclic factors chosen to appear in the presentation of $G$. Hence, it is an invariant of the pair $\left(G,\left(r_{1}, \ldots, r_{k}\right)\right)$ where $G=\mathbb{Z}_{r_{1}} \times \cdots \times \mathbb{Z}_{r_{k}}$.

The paper is organized as follows. In Section 2, we give the needed definitions. In Section 3, we define the statistics on $G_{r_{1}, \ldots, r_{k} ; n}$. Section 4 deals with the proof of Theorem 1.1. Section 5 deals with derangements in $G_{r_{1}, \ldots, r_{k} ; n}$ and the proof of Theorem 1.2. In Section 6 , we deal with the set of involutions in $G_{r_{1}, \ldots, r_{k} ; n}$ and the proof of Theorem 1.4. 


\section{The group of multi-colored permutations}

Definition 2.1. Let $r_{1}, \ldots, r_{k}$ and $n$ be positive integers. The group of multi-colored permutations of $n$ digits is the wreath product

$$
G_{r_{1}, \ldots, r_{k} ; n}=\left(\mathbb{Z}_{r_{1}} \times \mathbb{Z}_{r_{2}} \times \cdots \times \mathbb{Z}_{r_{k}}\right) \curlywedge S_{n}=\left(\mathbb{Z}_{r_{1}} \times \mathbb{Z}_{r_{2}} \times \cdots \times \mathbb{Z}_{r_{k}}\right)^{n} \rtimes S_{n},
$$

consisting of all the pairs $(Z, \tau)$ where $Z=\left(z_{i}^{j}\right)(1 \leq i \leq n, 1 \leq j \leq k)$ is an $n \times k$ matrix such that the elements of column $j(1 \leq j \leq k)$ belong to $\mathbb{Z}_{r_{j}}$ and $\tau \in S_{n}$. The multiplication is defined by the following rule: Let $Z, U$ be two $n \times k$ matrices as above and let $\sigma, \tau \in S_{n}$. Then

$$
(Z, \tau) \cdot(U, \sigma)=\left(\left(z_{i}^{j}+u_{\tau^{-1}(i)}^{j}\right), \tau \circ \tau^{\prime}\right)
$$

(here, in each column $j$, the + is taken modulo $r_{j}$ ).

Example 2.2. Let $r_{1}=3, r_{2}=2, r_{3}=2, r_{4}=3$ and $n=3$. Define

$$
\pi_{1}=\left(Z_{1}, \tau_{1}\right)=\left(\left(\begin{array}{llll}
0 & 1 & 0 & 2 \\
2 & 0 & 1 & 2 \\
1 & 1 & 0 & 1
\end{array}\right),\left(\begin{array}{lll}
1 & 2 & 3 \\
3 & 2 & 1
\end{array}\right)\right)
$$

and

$$
\pi_{2}=\left(Z_{2}, \tau_{2}\right)=\left(\left(\begin{array}{llll}
0 & 0 & 1 & 0 \\
0 & 1 & 1 & 1 \\
2 & 1 & 0 & 2
\end{array}\right),\left(\begin{array}{lll}
1 & 2 & 3 \\
2 & 3 & 1
\end{array}\right)\right) .
$$

Then we have:

$$
\begin{aligned}
& \pi_{1} \cdot \pi_{2}=\left(\left(\begin{array}{llll}
2 & 0 & 0 & 2 \\
1 & 0 & 1 & 2 \\
2 & 0 & 0 & 1
\end{array}\right),\left(\begin{array}{lll}
1 & 2 & 3 \\
2 & 1 & 3
\end{array}\right)\right) \\
& \pi_{2} \cdot \pi_{1}=\left(\left(\begin{array}{llll}
2 & 0 & 0 & 1 \\
2 & 1 & 0 & 0 \\
1 & 1 & 1 & 1
\end{array}\right),\left(\begin{array}{lll}
1 & 2 & 3 \\
1 & 3 & 2
\end{array}\right)\right) .
\end{aligned}
$$

Here is another description of the group $G_{r_{1}, \ldots, r_{k} ; n}$. Consider the alphabet

$$
\Sigma=\left\{i^{\left[z_{i}^{1}, \ldots, z_{i}^{k}\right]} \mid z_{i}^{j} \in \mathbb{Z}_{r_{j}}, 1 \leq i \leq n, 1 \leq j \leq k\right\} .
$$

The set $\Sigma$ can be seen as the set $[n]=\{1, \ldots, n\}$, colored by $k$ palettes of colors, the palette numbered $j$ having $r_{j}$ colors.

If we denote by $\theta_{j}$ the cyclic operator which colors the digit $i$ by first color from the $j$-th palette, then an element of $G_{r_{1}, \ldots, r_{k} ; n}$ is a multi-colored permutation, i.e. a bijection $\pi: \Sigma \rightarrow \Sigma$ such that

$$
\pi\left(\left(\theta_{1}^{\epsilon_{1}} \circ \theta_{2}^{\epsilon_{2}} \circ \cdots \circ \theta_{k}^{\epsilon_{k}}\right)(i)\right)=\left(\theta_{1}^{\epsilon_{1}} \circ \theta_{2}^{\epsilon_{2}} \circ \cdots \circ \theta_{k}^{\epsilon_{k}}\right)(\pi(i))
$$


where $\epsilon_{i} \in\{0,1\}, 1 \leq i \leq k$.

In particular, if $k=1$ we get the group $G_{r_{1}, n}=C_{r_{1}} 2 S_{n}$. This case has several subcases, for example if we take $r=r_{1}=1$, then we get the symmetric group $S_{n}$, while $r=2$ yields the hyperoctahedral group $B_{n}$, i.e., the classical Coxeter group of type $B$.

Here is an algebraic description of $G_{r_{1}, \ldots, r_{k} ; n}$. Define the following set of generators: $T=\left\{t_{1}, t_{2}, \ldots, t_{k}, s_{1}, \ldots, s_{n-1}\right\}$ with the following relations:

- $t_{i}^{r_{i}}=1,(i \in\{1, \ldots, k\})$

- $t_{i} t_{j}=t_{j} t_{i},(i, j \in\{1, \ldots, k\})$

- $\left(t_{i} s_{1}\right)^{2 r_{i}}=1,(i \in\{1, \ldots, k\})$

- $\left(t_{i} t_{j} s_{1}\right)^{2 r_{i} r_{j}}=1,(1 \leq i<j \leq k)$

- $s_{i}^{2}=1,(i \in\{1, \ldots, n-1\})$

- $s_{i} s_{j} s_{i}=s_{j} s_{i} s_{j},(1 \leq i<j<n, j-i=1)$

- $s_{i} s_{j}=s_{j} s_{i},(1 \leq i<j<n, j-i>1)$

- $t_{i} s_{j}=s_{j} t_{i},(1 \leq i \leq k, 1<j<n)$.

Realizing $t_{i}(1 \leq i \leq k)$ as the multi-colored permutation taking 1 to $1^{\overrightarrow{e_{i}}}$ (where $\vec{e}_{i}$ is the $i$-th standard vector) fixing pointwise the other digits, and $s_{i}$ as the adjacent Coxeter transposition $(i, i+1)(1 \leq i<n)$, it is easy to see that $G_{r_{1}, \ldots, r_{k} ; n}$ is actually the group generated by $T$ subject to the above relations. A Dynkin-type diagram for $G_{r_{1}, \ldots, r_{k} ; n}$ is presented in Figure 1.

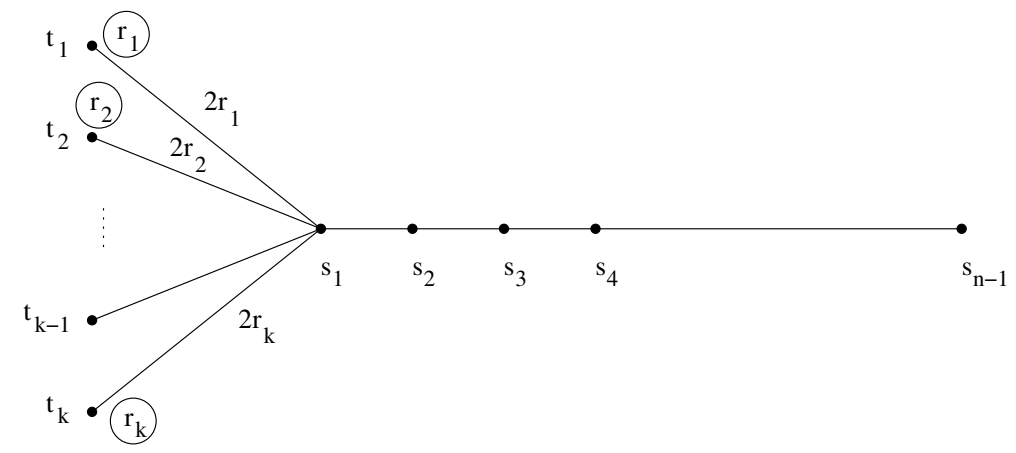

Figure 1: The "Dynkin diagram" of $G_{r_{1}, \ldots, r_{k} ; n}$ 


\section{Statistics on $G_{r_{1}, \ldots, r_{k} ; n}$}

We start by defining an order on the set:

$$
\Sigma=\left\{i^{\left(z_{i}^{1}, \ldots, z_{i}^{k}\right)} \mid z_{i}^{j} \in \mathbb{Z}_{r_{k}}, 1 \leq i \leq n, 1 \leq j \leq k\right\} .
$$

Define $r_{\max }=\max \left\{r_{1}, \ldots, r_{k}\right\}$. For any two vectors

$$
\vec{v}=\left(v_{1}, \ldots, v_{k}\right), \vec{w}=\left(w_{1}, \ldots, w_{k}\right) \in \mathbb{Z}_{r_{1}} \times \cdots \times \mathbb{Z}_{r_{k}},
$$

we write $\vec{v} \prec \vec{w}$ if

$$
w_{1} \cdot r_{\max }^{k-1}+\cdots+w_{k-1} \cdot r_{\max }+w_{k}<v_{1} \cdot r_{\max }^{k-1}+\cdots+v_{k-1} \cdot r_{\max }+v_{k} .
$$

For example, if $r_{1}=r_{2}=r_{3}=3$ then $(2,0,1) \prec(1,1,0)$.

We also write $i^{\vec{v}} \prec j^{\vec{w}}$ if:

1. $\vec{v} \neq \vec{w}$ and $\vec{v} \prec \vec{w}$, or

2. $\vec{v}=\vec{w}$ and $i<j$.

Based on this order, we define the excedance set of a permutation $\pi$ on $\Sigma$ :

$$
\operatorname{Exc}(\pi)=\{i \in \Sigma \mid \pi(i) \succ i\}
$$

and the excedance number is defined to be $\operatorname{exc}(\pi)=|\operatorname{Exc}(\pi)|$.

For simplifying the computations, we define the excedance number in a different way. The set $\Sigma$ can be divided into layers, according to the palettes. Explicitly, for each $\vec{v} \in \mathbb{Z}_{r_{1}} \times \cdots \times \mathbb{Z}_{r_{k}}$, define the layer $\Sigma^{\vec{v}}=\left\{1^{\vec{v}}, \ldots, n^{\vec{v}}\right\}$. We call the layer $\Sigma^{\overrightarrow{0}}$ the principal part of $\Sigma$. We will show that $\operatorname{exc}(\pi)$ can be computed using parameters defined only on $\Sigma^{\overrightarrow{0}}$.

Let $\pi=\left(\sigma,\left(z_{1}^{1}, \ldots, z_{1}^{k}\right),\left(z_{2}^{1}, \ldots, z_{2}^{k}\right), \ldots,\left(z_{n}^{1}, \ldots, z_{n}^{k}\right)\right) \in G_{r_{1}, \ldots, r_{k} ; n}$ and let $1 \leq p \leq k$. Define:

$$
\operatorname{csum}_{p}(\pi)=\sum_{i=1}^{n} z_{i}^{p} \cdot \prod_{t=1}^{p-1} \chi\left(z_{i}^{t}=0\right)
$$

where $\chi(P)$ is 1 if the property $P$ holds and 0 otherwise.

The parameter $\operatorname{csum}_{p}(\pi)$ sums the colors of palette $p$ where a color of a digit is counted only if there are no colors of preceding palettes on this digit.

Here is an easier way to understand the parameters $\operatorname{csum}_{p}(\pi)$ :

For $\pi=\left(\sigma,\left(z_{1}^{1}, \ldots, z_{1}^{k}\right),\left(z_{2}^{1}, \ldots, z_{2}^{k}\right), \ldots,\left(z_{n}^{1}, \ldots, z_{n}^{k}\right)\right) \in G_{r_{1}, \ldots, r_{k} ; n}$, write the $n \times k$ matrix $Z=\left(z_{i}^{j}\right)$. Then, $\operatorname{csum}_{p}$ is just the sum of the elements of the $p$-th column where we are ignoring the elements which are not leading in their rows. 
Example 3.1. Let

$$
\pi=\left(\left(\begin{array}{lll}
1 & 2 & 3 \\
3 & 1 & 2
\end{array}\right),(1,2,0,1),(0,0,1,2),(0,2,1,1)\right) \in G_{2,3,2,3 ; 3} .
$$

Then $Z=\left(\begin{array}{llll}\mathbf{1} & 2 & 0 & 1 \\ 0 & 0 & \mathbf{1} & 2 \\ 0 & \mathbf{2} & 1 & 1\end{array}\right)$

and thus we have:

$$
\operatorname{csum}_{1}(\pi)=1, \operatorname{csum}_{2}(\pi)=2, \operatorname{csum}_{3}(\pi)=1, \operatorname{csum}_{4}(\pi)=0 .
$$

Now define:

$$
\operatorname{Exc}_{A}(\pi)=\{i \in[n-1] \mid \pi(i) \succ i\} \text { and } \operatorname{exc}_{A}(\pi)=\left|\operatorname{Exc}_{A}(\pi)\right| .
$$

Proposition 3.2. Let $\pi=(Z, \sigma)$. Write $r=\prod_{j=1}^{k} r_{j}$. Then:

$$
\operatorname{exc}(\pi)=r \cdot \operatorname{exc}_{A}(\pi)+\sum_{p=1}^{k}\left(\operatorname{csum}_{p}(\pi) \cdot \prod_{q=1, q \neq p}^{k} r_{q}\right)
$$

Proof. Let $i \in[n]$. Write $\pi\left(i^{\overrightarrow{0}}\right)=j^{\vec{z}_{i}}$. We divide our treatment according to $\vec{z}_{i}=$ $\left(z_{i}^{1}, \ldots, z_{i}^{k}\right)$.

- $\vec{z}_{i}=\overrightarrow{0}$ : In this case, $i \in \operatorname{Exc}_{A}(\pi)$ if and only if $\sigma(i)>i$ or in other words: $\pi\left(i^{\overrightarrow{0}}\right) \succ i^{\overrightarrow{0}}$. This happens, if and only if, for each $\vec{\alpha}=\left(\alpha^{1}, \ldots, \alpha^{k}\right)$ where $0 \leq \alpha^{t} \leq r_{t}-1$, we have $\pi\left(i^{\vec{\alpha}}\right) \succ i^{\vec{\alpha}}$. Thus $i$ contributes $\prod_{j=1}^{k} r_{j}=r$ to $\operatorname{exc}(\pi)$.

- $\vec{z}_{i}=\left(z_{i}^{1}, \ldots, z_{i}^{k}\right) \neq \overrightarrow{0}$. In this case $i=i^{\overrightarrow{0}} \notin \operatorname{Exc}(\pi)$. We check now for which $\vec{v}$, $i^{\vec{v}} \in \operatorname{Exc}(\pi)$. Since $\pi\left(i^{\overrightarrow{0}}\right)=j^{\overrightarrow{z_{i}}}$, we have $\pi\left(i^{\vec{v}}\right)=j \vec{v}+\overrightarrow{z_{i}}$. Let $m \in\{1, \ldots, k\}$ be the minimal index such that $z_{i}^{m} \neq 0$ and $z_{i}^{t}=0$ for all $t<m$. Note that for all $\overrightarrow{0} \succ \vec{v} \succ\left(0, \ldots, 0, r_{m}-z_{i}^{m}, 0, \ldots, 0\right), \pi\left(i^{\vec{v}}\right)=j^{\vec{v}+\vec{z}_{i}} \prec i^{\vec{v}}$, hence $i^{\vec{v}} \notin \operatorname{Exc}(\pi)$.

Now, for all

$$
\left(0, \ldots, 0, r_{m}-z_{i}^{m}, 0, \ldots, 0\right) \succ \vec{v} \succ\left(0, \ldots, 0, r_{m}-1, r_{m+1}-1, \ldots, r_{k}-1\right),
$$

$\pi\left(i^{\vec{v}}\right)=j^{\vec{v}+z_{i}} \succ i^{\vec{v}}$, and hence $i^{\vec{v}} \in \operatorname{Exc}(\pi)$. So, it contributes $z_{i}^{m} \cdot r_{m+1} \cdots r_{k}$ elements to the excedance set.

In the same way, for each $\vec{w}=\left(\alpha_{1}, \ldots, \alpha_{m-1}, 0, \ldots, 0\right) \neq \overrightarrow{0}$ and for all

$$
\left(0, \ldots, 0, r_{m}-z_{i}^{m}, 0, \ldots, 0\right) \succ \vec{v} \succ\left(0, \ldots, 0, r_{m}-1, r_{m+1}-1, \ldots, r_{k}-1\right),
$$


$\pi\left(i^{i \vec{w}+\vec{v}}\right)=j^{\vec{w}+\vec{v}+z_{i}} \succ i^{\vec{w}+\vec{v}}$, and hence $i^{\vec{w}+\vec{v}} \in \operatorname{Exc}(\pi)$. So it contributes $\left(r_{1} \cdots r_{m-1}-\right.$ 1) $\cdot z_{i}^{m} \cdot r_{m+1} \cdots r_{k}$ elements to the excedance set.

Hence, this $i$ contributes

$$
r_{1} \cdots r_{m-1} \cdot z_{i}^{m} \cdot r_{m+1} \cdots r_{k}=z_{i}^{m} \prod_{q=1, q \neq m}^{k} r_{q} .
$$

Now, we sum the contributions over all $i \in\{1,2, \ldots, n\}$. Since we have $\operatorname{exc}_{A}(\pi)$ digits which satisfy $\vec{z}_{i}=\overrightarrow{0}$ and $\sigma(i)>i$, their total contribution is $r \cdot \operatorname{exc}_{A}(\pi)$, which is the first summand of $\operatorname{exc}(\pi)$.

The other digits have $\vec{z}_{i} \neq \overrightarrow{0}$, so their contribution is

$$
\begin{aligned}
\sum_{\left\{i \mid \vec{z}_{i} \neq \overrightarrow{0}\right\}}\left(z_{i}^{m} \prod_{q=1, q \neq m}^{k} r_{q}\right) & =\sum_{p=1}^{k}\left(\left(\sum_{i=1}^{n} z_{i}^{p} \cdot \prod_{t=1}^{p-1} \chi\left(z_{i}^{t}=0\right)\right) \prod_{q=1, q \neq p}^{k} r_{q}\right) \\
& =\sum_{p=1}^{k}\left(\operatorname{csum}_{p}(\pi) \cdot \prod_{q=1, q \neq p}^{k} r_{q}\right),
\end{aligned}
$$

which is the second summand of $\operatorname{exc}(\pi)$, and hence we are done.

Example 3.3. Let

$$
\pi=\left(\begin{array}{ccc}
1 & 2 & 3 \\
3^{(0,0)} & 1^{(2,1)} & 2^{(0,1)}
\end{array}\right)=\left(\left(\begin{array}{ll}
0 & 0 \\
\mathbf{2} & 1 \\
0 & \mathbf{1}
\end{array}\right),\left(\begin{array}{lll}
1 & 2 & 3 \\
3 & 1 & 2
\end{array}\right)\right) \in G_{3,2 ; 3} .
$$

We write $\pi$ in its extended form:

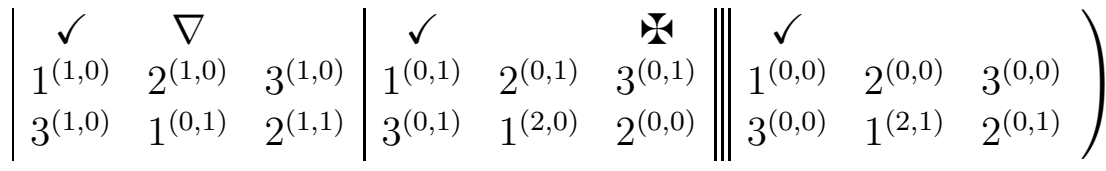

$$
\begin{aligned}
& \left(\begin{array}{ccc|ccc|ccc|}
\checkmark & \nabla & \mathbf{v} & \checkmark & \nabla & & \checkmark & \nabla & \mathbf{v} \\
1^{(2,1)} & 2^{(2,1)} & 3^{(2,1)} & 1^{(2,0)} & 2^{(2,0)} & 3^{(2,0)} & 1^{(1,1)} & 2^{(1,1)} & 3^{(1,1)} \\
3^{(2,1)} & 1^{(1,0)} & 2^{(2,0)} & 3^{(2,0)} & 1^{(1,1)} & 2^{(2,1)} & 3^{(1,1)} & 1^{(0,0)} & 2^{(1,0)}
\end{array}\right.
\end{aligned}
$$

We have $\operatorname{exc}(\pi)=13$, while $\operatorname{csum}_{1}(\pi)=2$ and $\operatorname{csum}_{2}(\pi)=1$.

Recall that any permutation of $S_{n}$ can be decomposed into a product of disjoint cycles. This notion can be easily generalized to the group $G_{r_{1}, \ldots, r_{k} ; n}$ as follows. Given any $\pi \in G_{r_{1}, \ldots, r_{k} ; n}$ we define the cycle number of $\pi=(Z, \sigma)$ to be the number of cycles in $\sigma$.

We say that $i \in[n]$ is an absolute fixed point of $\pi \in G_{r_{1}, \ldots, r_{k} ; n}$ if $\sigma(i)=i$. 


\section{Proof of Theorem 1.1}

In this section we prove Theorem 1.1. The way to prove this type of identities is to construct a subset $S$ of $G_{r_{1}, \ldots, r_{k} ; n}$ whose contribution to the generating function is exactly the right side of the identity. Then, we have to construct a killing involution on $G_{r_{1}, \ldots, r_{k} ; n}-$ $S$, i.e., an involution on $G_{r_{1}, \ldots, r_{k} ; n}-S$ which preserves the number of excedances but changes the sign of every element of $G_{r_{1}, \ldots, r_{k} ; n}-S$ and hence shows that $G_{r_{1}, \ldots, r_{k} ; n}-S$ contributes nothing to the generating function.

Recall that $r=r_{1} \cdots r_{k}$. We divide $G_{r_{1}, \ldots, r_{k} ; n}$ into $2 r+1$ disjoint subsets as follows:

$$
\begin{gathered}
K=\left\{\pi \in G_{r_{1}, \ldots, r_{k} ; n}|| \pi(n)|\neq n,| \pi(n-1) \mid \neq n\right\}, \\
T_{n}^{\vec{v}}=\left\{\pi \in G_{r_{1}, \ldots, r_{k} ; n} \mid \pi(n)=n^{\vec{v}}\right\}, \quad\left(\vec{v} \in \mathbb{Z}_{r_{1}} \times \cdots \times \mathbb{Z}_{r_{k}}\right), \\
R_{n}^{\vec{v}}=\left\{\pi \in G_{r_{1}, \ldots, r_{k} ; n} \mid \pi(n-1)=n^{\vec{v}}\right\}, \quad\left(\vec{v} \in \mathbb{Z}_{r_{1}} \times \cdots \times \mathbb{Z}_{r_{k}}\right),
\end{gathered}
$$

We first construct a killing involution on the set $K$. Let $\pi \in K$. Define $\varphi: K \rightarrow K$ by

$$
\pi^{\prime}=\varphi(\pi)=(\pi(n-1), \pi(n)) \pi .
$$

Note that $\varphi$ exchanges $\pi(n-1)$ with $\pi(n)$. It is obvious that $\varphi$ is indeed an involution.

We will show that $\operatorname{exc}(\pi)=\operatorname{exc}\left(\pi^{\prime}\right)$. First, for $i<n-1$, it is clear that $i \in \operatorname{Exc}(\pi)$ if and only if $i \in \operatorname{Exc}\left(\pi^{\prime}\right)$. Now, as $\pi(n-1) \neq n, n-1 \notin \operatorname{Exc}(\pi)$ and thus $n \notin \operatorname{Exc}\left(\pi^{\prime}\right)$. Finally, $\pi(n) \neq n$ implies that $n-1 \notin \operatorname{Exc}\left(\pi^{\prime}\right)$ and thus $\operatorname{exc}(\pi)=\operatorname{exc}\left(\pi^{\prime}\right)$.

On the other hand, $\operatorname{cyc}(\pi)$ and $\operatorname{cyc}\left(\pi^{\prime}\right)$ have different parities due to a multiplication by a transposition. Hence, $\varphi$ is indeed a killing involution on $K$.

We turn now to the sets $T_{n}^{\vec{v}}\left(\vec{v}=\left(z_{n}^{1}, \ldots, z_{n}^{k}\right) \in \mathbb{Z}_{r_{1}} \times \cdots \times \mathbb{Z}_{r_{k}}\right)$. Note that there is a natural bijection between $T_{n}^{\vec{v}}$ and $G_{r_{1}, \ldots, r_{k} ; n-1}$ defined by ignoring the last digit. Let $\pi \in T_{n}^{\vec{v}}$. Denote the image of $\pi \in T_{n}^{\vec{v}}$ under this bijection by $\pi^{\prime}$. Since $n \notin \operatorname{Exc}_{\mathrm{A}}(\pi)$, we have $\operatorname{exc}_{\mathrm{A}}(\pi)=\operatorname{exc}_{\mathrm{A}}\left(\pi^{\prime}\right)$.

Let $m \in\{1, \ldots, k\}$ be the minimal index such that $z_{n}^{m} \neq 0$ and $z_{n}^{t}=0$ for all $t<m$. Then, $\operatorname{csum}_{m}\left(\pi^{\prime}\right)=\operatorname{csum}_{m}(\pi)-z_{n}^{m}$, and $\operatorname{csum}_{p}\left(\pi^{\prime}\right)=\operatorname{csum}_{\mathrm{p}}(\pi)$ for $1 \leq p \leq k, p \neq m$. Finally, since $n$ is an absolute fixed point of $\pi, \operatorname{cyc}\left(\pi^{\prime}\right)=\operatorname{cyc}(\pi)-1$. Hence, we get that the total contribution of $T_{n}^{\vec{v}}$ is:

$$
P_{T_{n}^{\vec{v}}}=-q^{z_{n}^{m}} \prod_{q=1, q \neq m}^{k} r_{q} P_{G_{r_{1}, \ldots, r_{k} ; n-1}}(q, 1,-1)=-q^{z_{n}^{m} \frac{r}{r_{m}}} P_{G_{r_{1}, \ldots, r_{k} ; n-1}}(q, 1,-1)
$$

where $m$ is defined as above.

Now, we treat the sets $R_{n}^{\vec{v}} \quad\left(\vec{v}=\left(z_{n}^{1}, \ldots, z_{n}^{k}\right) \in \mathbb{Z}_{r_{1}} \times \cdots \times \mathbb{Z}_{r_{k}}\right)$. There is a bijection between $R_{n}^{\vec{v}}$ and $T_{n}^{\vec{v}}$ using the same function $\varphi$ we used above. Let $\pi \in R_{n}^{\vec{v}}$. Define $\varphi: R_{n}^{\vec{v}} \rightarrow T_{n}^{\vec{v}}$ by

$$
\pi^{\prime}=\varphi(\pi)=(\pi(n-1), \pi(n)) \pi
$$


When we compute the change in the excedance, we split our treatment into two cases: $\vec{v}=\overrightarrow{0}$ and $\vec{v} \neq \overrightarrow{0}$.

We start with the case $\vec{v}=\overrightarrow{0}$. Note that $n-1 \in \operatorname{Exc}_{\mathrm{A}}(\pi)$ (since $\pi(n-1)=n$ ) and $n \notin \operatorname{Exc}_{\mathrm{A}}(\pi)$. On the other hand, in $\pi^{\prime}, n-1, n \notin \operatorname{Exc}_{\mathrm{A}}\left(\pi^{\prime}\right)$. Hence, $\operatorname{exc}_{\mathrm{A}}(\pi)-1=\operatorname{exc}_{\mathrm{A}}\left(\pi^{\prime}\right)$.

Now, for the case $\vec{v} \neq \overrightarrow{0}: n-1, n \notin \operatorname{Exc}_{\mathrm{A}}(\pi)$ (since $\pi(n-1)=n^{\vec{v}}$ is not an excedance). We also have: $n-1, n \notin \operatorname{Exc}_{\mathrm{A}}\left(\pi^{\prime}\right)$ and thus $\operatorname{Exc}_{\mathrm{A}}(\pi)=\operatorname{Exc}_{\mathrm{A}}\left(\pi^{\prime}\right)$ for $\pi \in R_{n}^{\vec{v}}$ where $\vec{v} \neq \overrightarrow{0}$.

In both cases, we have that $\operatorname{csum}_{\mathrm{p}}(\pi)=\operatorname{csum}_{\mathrm{p}}\left(\pi^{\prime}\right)$ for each $1 \leq p \leq k$. Hence, we have that $\operatorname{exc}(\pi)-r=\operatorname{exc}\left(\pi^{\prime}\right)$ for $\vec{v}=\overrightarrow{0}$ and $\operatorname{exc}(\pi)=\operatorname{exc}\left(\pi^{\prime}\right)$ for $\vec{v} \neq \overrightarrow{0}$.

As before, the number of cycles changes its parity due to the multiplication by a transposition, and hence: $(-1)^{\operatorname{cyc}(\pi)}=-(-1)^{\operatorname{cyc}\left(\pi^{\prime}\right)}$.

Hence, the total contribution of the elements in $R_{n}^{\vec{v}}$ is

$$
q^{r} P_{G_{r_{1}, \ldots, r_{k} ; n-1}}(q, 1,-1)
$$

for $\vec{v}=\overrightarrow{0}$, and

$$
q^{z_{n}^{m}} \prod_{q=1, q \neq m}^{k}{ }^{r_{q}} P_{G_{r_{1}, \ldots, r_{k} ; n-1}}(q, 1,-1)=q^{z_{n}^{m} \frac{r}{r_{m}}} P_{G_{r_{1}, \ldots, r_{k} ; n-1}}(q, 1,-1)
$$

for $\vec{v} \neq \overrightarrow{0}$.

In order to calculate $\sum_{\vec{v} \in \mathbb{Z}_{r_{1}} \times \cdots \times \mathbb{Z}_{r_{k}}} P_{T_{n}^{\vec{v}}}$ and $\sum_{\vec{v} \in \mathbb{Z}_{r_{1}} \times \cdots \times \mathbb{Z}_{r_{k}}} P_{R_{n}^{\vec{v}}}$, we have to divide $\mathbb{Z}_{r_{1}} \times$ $\cdots \times \mathbb{Z}_{r_{k}}$ into sets according to the minimal index $m$ such that $z_{n}^{m} \neq 0$ and $z_{n}^{t}=0$ for all $t<m$.

For each $m \in\{1, \ldots, k+1\}$, denote:

$$
W_{m}=\left\{\vec{v}=\left(z_{n}^{1}, \ldots, z_{n}^{k}\right) \in \mathbb{Z}_{r_{1}} \times \cdots \times \mathbb{Z}_{r_{k}} \mid z_{n}^{m} \neq 0, z_{n}^{t}=0, \forall t<m\right\} .
$$

Note that $W_{k+1}=\{\overrightarrow{0}\}$.

It is easy to see that $\left\{W_{1}, \ldots, W_{k}, W_{k+1}\right\}$ is a partition of $\mathbb{Z}_{r_{1}} \times \cdots \times \mathbb{Z}_{r_{k}}$.

Hence

$$
\sum_{\vec{v} \in \mathbb{Z}_{r_{1}} \times \cdots \times \mathbb{Z}_{r_{k}}} P_{T_{n}^{\vec{n}}}=\sum_{m=1}^{k+1}\left(\sum_{\vec{v} \in W_{m}} P_{T_{n}^{\vec{n}}}\right)=\left(-1+\sum_{m=1}^{k} r_{m+1} \cdots r_{k} \sum_{t=1}^{r_{m}-1}-q^{t \frac{r}{r_{m}}}\right) P_{G_{r_{1}, \ldots, r_{k} ; n-1}}(q, 1,-1) .
$$

Similarly, we get:

$$
\sum_{\vec{v} \in \mathbb{Z}_{r_{1}} \times \cdots \times \mathbb{Z}_{r_{k}}} P_{R_{n}^{\vec{n}}}=\left(q^{r}+\sum_{m=1}^{k} r_{m+1} \cdots r_{k} \sum_{t=1}^{r_{m}-1} q^{t \frac{r}{r_{m}}}\right) P_{G_{r_{1}, \ldots, r_{k} ; n-1}}(q, 1,-1) .
$$

Now, if we sum up all the parts, we get:

$$
\begin{aligned}
P_{G_{r_{1}, \ldots, r_{k} ; n}}(q, 1,-1) & =\sum_{\vec{v} \in \mathbb{Z}_{r_{1}} \times \cdots \times \mathbb{Z}_{r_{k}}} P_{T_{n}^{\vec{v}}}+\sum_{\vec{v} \in \mathbb{Z}_{r_{1}} \times \cdots \times \mathbb{Z}_{r_{k}}} P_{R_{n}^{\vec{v}}} \\
& =\left(q^{r}-1\right) P_{G_{r_{1}, \ldots, r_{k} ; n-1}}(q, 1,-1)
\end{aligned}
$$


as needed.

From now on, we denote

$$
K(q)=K\left(q ; r_{1}, \ldots, r_{k}\right)=\sum_{m=1}^{k} r_{m+1} \cdots r_{k} \sum_{t=1}^{r_{m}-1} q^{t \frac{r}{r_{m}}}
$$

Note that:

$$
1+K(q)=\sum_{\vec{v} \in \mathbb{Z}_{r_{1}} \times \cdots \times \mathbb{Z}_{r_{k}}} q^{\operatorname{exc}\left(1^{\vec{v}}\right)}
$$

Now, for $n=1, G_{r_{1}, \ldots, r_{k} ; 1}$ is $\mathbb{Z}_{r_{1}} \times \cdots \times \mathbb{Z}_{r_{k}}$, and thus

$$
P_{G_{r_{1}, \ldots, r_{k} ; 1}}(q, 1,-1)=-1-K(q) .
$$

Hence, we have

$$
P_{G_{r_{1}, \ldots, r_{k} ; n}}(q, 1,-1)=(-1-K(q))\left(q^{r}-1\right)^{n-1},
$$

and we have finished the proof of Theorem 1.1.

\section{Derangements in $G_{r_{1}, \ldots, r_{k} ; n}$ and the proof of Theorem 1.2}

We start with the definition of a derangement.

Definition 5.1. An element $\sigma \in G_{r_{1}, \ldots, r_{k} ; n}$ is called a derangement if it has no absolute fixed points, i.e. $|\pi(i)| \neq i$ for every $i \in[n]$. Denote by $D_{r_{1}, \ldots, r_{k} ; n}$ the set of all derangements in $G_{r_{1}, \ldots, r_{k} ; n}$.

We prove now Theorem 1.2.

We divide $D_{r_{1}, \ldots, r_{k} ; n}$ into $r+2=r_{1} r_{2} \cdots r_{k}+2$ disjoint subsets in the following way:

$$
\begin{gathered}
A_{r_{1}, \ldots, r_{k} ; n}^{\vec{v}}=\left\{\pi \in D_{r_{1}, \ldots, r_{k} ; n}\left|\pi(2)=1^{\vec{v}},\right| \pi(1) \mid \neq 2\right\}, \vec{v} \in \mathbb{Z}_{r_{1}} \times \cdots \times \mathbb{Z}_{r_{k}} . \\
B_{r_{1}, \ldots, r_{k} ; n}=\left\{\pi \in D_{r_{1}, \ldots, r_{k} ; n}|| \pi \mid=(123 \cdots n)\right\} . \\
\hat{D}_{r_{1}, \ldots, r_{k} ; n}=D_{r_{1}, \ldots, r_{k} ; n}-\left(\bigcup_{\vec{v} \in \mathbb{Z}_{r_{1}} \times \cdots \times \mathbb{Z}_{r_{k}}} A_{r_{1}, \ldots, r_{k} ; n}^{\vec{v}} \cup B_{r_{1}, \ldots, r_{k} ; n}\right) .
\end{gathered}
$$

We start by constructing a killing involution $\varphi$ on $\hat{D}_{r_{1}, \ldots, r_{k} ; n}$. Given any $\pi \in \hat{D}_{r_{1}, \ldots, r_{k} ; n}$, let $i$ be the first number such that $|\pi(i)| \neq i+1$. Define

$$
\pi^{\prime}=\varphi(\pi)=(\pi(i), \pi(i+1)) \pi
$$


It is easy to see that $\varphi$ is a well-defined involution on $\hat{D}_{r_{1}, \ldots, r_{k} ; n}$. We proceed to prove that $\operatorname{exc}(\pi)=\operatorname{exc}\left(\pi^{\prime}\right)$. Indeed, $\operatorname{csum}_{p}(\pi)=\operatorname{csum}_{p}\left(\pi^{\prime}\right)$ for all $1 \leq p \leq k$.

Let $i$ be the first number such that $|\pi(i)| \neq i+1$, so that in the pass from $\pi$ to $\pi^{\prime}$ we exchange $\pi(i)$ with $\pi(i+1)$. For every $j \neq i, i+1$, clearly $j \in \operatorname{Exc}_{A}(\pi)$ if and only if $j \in \operatorname{Exc}_{A}\left(\pi^{\prime}\right)$. Since $\pi \in D_{r_{1}, \ldots, r_{k} ; n},|\pi(i)| \neq i+1$ and $|\pi(j)|=j+1$ for $j<i$, we have that $|\pi(i)|,|\pi(i+1)| \in\{1, i+2, \ldots, n\}$. Thus, exchanging $\pi(i)$ with $\pi(i+1)$ does not change $\operatorname{Exc}_{A}(\pi)$.

Note also that the parity of $\operatorname{cyc}\left(\pi^{\prime}\right)$ is opposite to the parity of $\operatorname{cyc}(\pi)$ due to the multiplication by a transposition. Hence, we have proven that $\varphi$ is indeed a killing involution.

Now, let us calculate the contribution of each set in our decomposition to

$$
P_{G_{r_{1}, \ldots, r_{k} ; n}}(q, 0,-1) \text {. }
$$

As we have shown, $\hat{D}_{r_{1}, \ldots, r_{k} ; n}$ contributes nothing.

Let $\vec{v}=\left(z_{2}^{1}, \ldots, z_{2}^{n}\right)$. Define a bijection

$$
\psi: A_{r_{1}, \ldots, r_{k} ; n}^{\vec{v}} \rightarrow D_{r_{1}, \ldots, r_{k} ; n-1}
$$

by: $\psi(\pi)=\pi^{\prime}$ where $\pi^{\prime}(1)=(|\pi(1)|-1)^{z_{1}(\pi)}$ and for $j>1, \pi^{\prime}(j)=(|\pi(j+1)|-1)^{z_{j+1}(\pi)}$. For example, if $\pi=\left(3^{(0,1,0)} 1^{(0,0,0)} 4^{(2,2,2)} 2^{(0,0,1)}\right)$, then $\pi^{\prime}=\left(2^{(0,1,0)} 3^{(2,2,2)} 1^{(0,0,1)}\right)$. It is easy to see that $\operatorname{exc}_{A}(\pi)=\operatorname{exc}_{A}\left(\pi^{\prime}\right)$. On the other hand, let $m \in\{1, \ldots, k\}$ be the minimal index such that $z_{2}^{m} \neq 0$ and $z_{n}^{t}=0$ for all $t<m$. Then $\operatorname{csum}_{m}\left(\pi^{\prime}\right)=\operatorname{csum}_{m}(\pi)-z_{2}^{m}$, and $\operatorname{csum}_{p}\left(\pi^{\prime}\right)=\operatorname{csum}_{p}(\pi)$ for $1 \leq p \leq k, p \neq m$.

We have also: $\operatorname{cyc}(\pi)=\operatorname{cyc}\left(\pi^{\prime}\right)$ and thus the contribution of $A_{r, n}^{\vec{v}}$ to $P_{G_{r_{1}, \ldots, r_{k} ; n}}(q, 0,-1)$ is

$$
P_{A_{r_{1}, \ldots, r_{k} ; n}}=q^{z_{2}^{m}} \prod_{q=1, q \neq m}^{k}{ }^{r_{q}} P_{G_{r_{1}, \ldots, r_{k} ; n-1}}(q, 0,-1)=q^{z_{2}^{m} \frac{r}{r_{m}}} P_{G_{r_{1}, \ldots, r_{k} ; n-1}}(q, 0,-1)
$$

where $m$ is defined as above.

Finally, we treat the set $B_{r_{1}, \ldots, r_{k} ; n}$. Here for every $\pi \in B_{r_{1}, \ldots, r_{k} ; n}$ we have $\operatorname{cyc}(\pi)=1$.

We calculate now:

$$
\begin{aligned}
P_{B_{r_{1}, \ldots, r_{k} ; n}}(q, 0,-1) & =-\prod_{s=1}^{n} \sum_{\vec{v}_{s} \in \mathbb{Z}_{r_{1} \times \cdots \times \mathbb{Z}_{r_{k}}}} q^{\operatorname{exc}\left(2^{\vec{v}_{1}} 3^{\left.\vec{v}_{2} \cdots n^{\vec{v}_{n}-1} 1^{\vec{v}_{n}}\right)}\right.} \\
& =-\left(q^{r}+K(q)\right)^{n-1} \cdot(1+K(q)) .
\end{aligned}
$$

To summarize, we get:

$$
\begin{aligned}
P_{G_{r_{1}, \ldots, r_{k} ; n}}(q, 0,-1) & =\left(\sum_{\vec{v}=\left(z_{2}^{1}, \ldots, z_{2}^{k}\right) \in \mathbb{Z}_{r_{1}} \times \cdots \times \mathbb{Z}_{r_{k}}} P_{A_{r_{1}, \ldots, r_{k} ; n}}\right)+P_{B_{r_{1}, \ldots, r_{k} ; n}} \\
& =\left(\sum_{m=1}^{k+1} \sum_{\vec{v} \in W_{m}} P_{A_{r_{1}, \ldots, r_{k} ; n}}\right)+P_{B_{r_{1}, \ldots, r_{k} ; n}} \\
& =(1+K(q)) P_{G_{r_{1}, \ldots, r_{k} ; n-1}}(q, 0,-1)-\left(q^{r}+K(q)\right)^{n-1} \cdot(1+K(q)) \\
& =(1+K(q))\left(P_{G_{r_{1}, \ldots, r_{k} ; n-1}}(q, 0,-1)-\left(q^{r}+K(q)\right)^{n-1}\right),
\end{aligned}
$$


so we get:

$$
P_{G_{r_{1}, \ldots, r_{k} ; n}}(q, 0,-1)=(1+K(q))\left(P_{G_{r_{1}, \ldots, r_{k} ; n-1}}(q, 0,-1)-\left(q^{r}+K(q)\right)^{n-1}\right) .
$$

Now, for $n=2$ we have:

$$
P_{G_{r_{1}, \ldots, r_{k} ; 2}}(q, 0,-1)=\left(q^{r}+K(q)\right) \cdot(1+K(q)) .
$$

By a direct computation, one can now get:

$$
P_{G_{r_{1}, \ldots, r_{k} ; n}}(q, 0,-1)=\left(q^{r}+K(q)\right)(1+K(q)) \cdot\left((1+K(q))^{n-2}-\sum_{k=1}^{n-2}\left(q^{r}+K(q)\right)^{k}(1+K(q))^{n-2-k}\right)
$$

for all $n \geq 2$ as in Theorem 1.2.

\section{Involutions in $G_{r_{1}, \ldots, r_{k} ; n}$}

We recall that an element $\sigma$ in $G_{r_{1}, \ldots, r_{k} ; n}$ is called an involution if $\sigma^{2}=1$. The set of involutions in $G_{r_{1}, \ldots, r_{k} ; n}$ will be denoted by $I_{r_{1}, \ldots, r_{k} ; n}$.

We consider the multi-distribution of the parameters exc, fix and csum on $I_{r_{1}, \ldots, r_{k} ; n}$, where $\operatorname{csum}(\pi)$ here is the total contribution of all the $\operatorname{csum}_{p}$-s from all the palettes:

$$
\operatorname{csum}(\pi)=\operatorname{exc}(\pi)-r \cdot \operatorname{exc}_{A}(\pi)=\sum_{p=1}^{k}\left(\operatorname{csum}_{p}(\pi) \cdot \prod_{q=1, q \neq p}^{k} r_{q}\right) .
$$

We start by classifying the involutions of $G_{r_{1}, \ldots, r_{k} ; n}$. As in the case of $G_{r, n}$, each involution of $G_{r_{1}, \ldots, r_{k} ; n}$ can be decomposed into a product of 'atomic' involutions of two types: absolute fixed points and 2-cycles.

An absolute fixed point must be of the form $\pi(i)=i^{\vec{v}}$ where $2 \vec{v}=\overrightarrow{0}$.

The 2-cycles have the form $\pi(i)=j^{\vec{v}_{1}} ; \pi(j)=i^{\vec{v}_{2}}$ where $\vec{v}_{1}, \vec{v}_{2} \in \mathbb{Z}_{1} \times \cdots \times \mathbb{Z}_{k}$ and $\vec{v}_{1}+\vec{v}_{2}=\overrightarrow{0}$.

Now, we compute recurrence and explicit formulas for

$$
f_{r_{1}, \ldots, r_{k} ; n}(u, v, w)=\sum_{\pi \in I_{r_{1}, \ldots, r_{k} ; n}} u^{\mathrm{fix}(\pi)} v^{\operatorname{exc}(\pi)} w^{\operatorname{csum}(\pi)} .
$$

Let $\pi$ be any involution in $I_{r_{1}, \ldots, r_{k} ; n}$. Then we have either $\pi(n)=n^{\vec{v}}$ or $\pi(n)=k^{\vec{v}}$ with $k<n$.

For $\pi \in I_{r_{1}, \ldots, r_{k} ; n}$ such that $\pi(n)=n^{\vec{v}}$, define $\pi^{\prime} \in I_{r_{1}, \ldots, r_{k} ; n-1}$ by ignoring the last digit of $\pi$. For $\pi \in I_{r_{1}, \ldots, r_{k} ; n}$ with $\pi(n)=k^{\vec{v}}$ and $\pi(k)=n^{\left(r_{1}, \ldots, r_{k}\right)-\vec{v}}$, define $\pi^{\prime \prime} \in I_{r_{1}, \ldots, r_{k} ; n-2}$ in the following way: Write $\pi$ in its complete notation, i.e. as a matrix of two rows. The first row of $\pi^{\prime \prime}$ is $(1,2, \ldots, n-2)$ while the second row is obtained from the second row of 
$\pi$ by ignoring the digits $n$ and $k$, and the other digits are placed in an order preserving way with respect to the second row of $\pi$. Here is an explicit formula for the map $\pi \mapsto \pi^{\prime \prime}$.

$$
\pi^{\prime \prime}(i)=\left\{\begin{array}{cccc}
\pi(i) & 1 \leq i<k & \text { and } & \pi(i)<k \\
\pi(i)-1 & 1 \leq i<k & \text { and } & \pi(i)>k \\
\pi(i-1) & k \leq i<n & \text { and } & \pi(i)<k \\
\pi(i-1)-1 & k \leq i<n & \text { and } & \pi(i)>k
\end{array}\right.
$$

Note that the map $\pi \mapsto \pi^{\prime}$ is a bijection from the set

$$
\left\{\pi \in I_{r_{1}, \ldots, r_{k} ; n} \mid \pi(n)=n^{\vec{v}}\right\} \quad(\vec{v} \text { fixed })
$$

to $I_{r_{1}, \cdots, r_{k} ; n-1}$, while $\pi \mapsto \pi^{\prime \prime}$ is a bijection from the set

$$
\left\{\pi \in I_{r_{1}, \ldots, r_{k} ; n} \mid \pi(n)=k^{\vec{v}}\right\} \quad(\vec{v} \text { fixed })
$$

to $I_{r_{1}, \ldots, r_{k} ; n-2}$.

For any $\vec{v}$, if $\pi(n)=n^{\vec{v}}$ then:

$$
\begin{aligned}
& \operatorname{fix}(\pi)=\operatorname{fix}\left(\pi^{\prime}\right)+1, \\
& \operatorname{exc}_{A}(\pi)=\operatorname{exc}_{A}\left(\pi^{\prime}\right) .
\end{aligned}
$$

Since $\vec{v}$ satisfies $2 \vec{v}=0$, we have two cases. If $\vec{v}=\overrightarrow{0}$, then:

$$
\operatorname{csum}(\pi)=\operatorname{csum}\left(\pi^{\prime}\right) .
$$

On the other hand, if $\vec{v}=\left(z_{1}, \ldots, z_{k}\right) \neq \overrightarrow{0}$, then there is some $m, 1 \leq m \leq k$, such that $z_{m} \neq 0$, and $z_{i}=0$ for all $1 \leq i<m$. In this case, $\vec{v}$ contributes $z_{m} \cdot \frac{r}{r_{m}}$ to $\operatorname{csum}(\pi)$. But since $\pi$ is an involution, we have $z_{m}=\frac{r_{m}}{2}$, and thus we have:

$$
\operatorname{csum}(\pi)=\operatorname{csum}\left(\pi^{\prime}\right)+\frac{r}{2} .
$$

If $\pi(n)=t^{\overrightarrow{v_{1}}}$, then the parameters satisfy

$$
\begin{gathered}
\operatorname{fix}(\pi)=\operatorname{fix}\left(\pi^{\prime \prime}\right), \\
\operatorname{exc}_{A}(\pi)=\operatorname{exc}_{A}\left(\pi^{\prime \prime}\right)+\delta_{\vec{v}, \overrightarrow{0}} .
\end{gathered}
$$

where $\delta_{\vec{v}_{1}, \vec{v}_{2}}$ is a generalized Kronecker Delta:

$$
\delta_{\vec{v}_{1}, \vec{v}_{2}}=\left\{\begin{array}{ll}
1 & \vec{v}_{1}=\vec{v}_{2} \\
0 & \vec{v}_{1} \neq \vec{v}_{2}
\end{array} .\right.
$$

Note that $\pi(t)=n^{\overrightarrow{v_{2}}}$, where $\vec{v}_{1}+\vec{v}_{2}=0$. Again, we have two cases. If $\vec{v}_{1}=\overrightarrow{0}$, then $\vec{v}_{2}=\overrightarrow{0}$, and:

$$
\operatorname{csum}(\pi)=\operatorname{csum}\left(\pi^{\prime}\right) .
$$


On the other hand, if $\vec{v}_{1}=\left(z_{1}, \ldots, z_{k}\right) \neq \overrightarrow{0}$, then there is some $m, 1 \leq m \leq k$, such that $z_{m} \neq 0$, and $z_{i}=0$ for all $1 \leq i<m$. Since $\vec{v}_{1}+\vec{v}_{2}=0$, we have that $\vec{v}_{2}=\left(z_{1}^{\prime}, \ldots, z_{k}^{\prime}\right) \neq \overrightarrow{0}$ with $z_{m}^{\prime} \neq 0, z_{i}^{\prime}=0$ for all $1 \leq i<m$, and $z_{m}+z_{m}^{\prime}=r_{m}$. Now, $\vec{v}_{1}$ contributes $z_{m} \cdot \frac{r}{r_{m}}$ to $\operatorname{csum}(\pi)$ while $\vec{v}_{2}$ contributes $z_{m}^{\prime} \cdot \frac{r}{r_{m}}$ to $\operatorname{csum}(\pi)$. Hence, their total contribution is:

$$
z_{m} \cdot \frac{r}{r_{m}}+z_{m}^{\prime} \cdot \frac{r}{r_{m}}=\left(z_{m}+z_{m}^{\prime}\right) \cdot \frac{r}{r_{m}}=r_{m} \cdot \frac{r}{r_{m}}=r
$$

Thus we have:

$$
\operatorname{csum}(\pi)=\operatorname{csum}\left(\pi^{\prime \prime}\right)+r\left(1-\delta_{\vec{v}, \overrightarrow{0}}\right)
$$

Now define

$$
\epsilon=\#\left\{r_{i} \mid 1 \leq i \leq k, \quad r_{i}=0 \quad(\bmod 2)\right\}
$$

Define also

$$
\mu=\mu_{r_{1}, \ldots, r_{k}}=\left\{\begin{array}{cc}
1+2^{\epsilon} w^{\frac{r}{2}} & \epsilon \neq 0 \\
1 & \epsilon=0
\end{array} .\right.
$$

The above consideration gives the following recurrence formula:

$$
\begin{aligned}
f_{r_{1}, \ldots, r_{k} ; n}(u, v, w)= & u \mu f_{r_{1}, \ldots, r_{k} ; n-1}(u, v, w) \\
& +(n-1)\left(v+(r-1) w^{r}\right) f_{r_{1}, \ldots, r_{k} ; n-2}(u, v, w), \quad n \geq 1 .
\end{aligned}
$$

Using the same technique used in [2], we get the following explicit formula:

Corollary 6.1. The polynomial $f_{r_{1}, \ldots, r_{k} ; n}(u, v, w)$ is given by

$$
\sum_{j=n / 2}^{n}(n-j) !\left(\begin{array}{c}
n \\
n-j, n-j, 2 j-n
\end{array}\right) \frac{u^{2 j-n}\left(v+(r-1) w^{r}\right)^{n-j}}{2^{n-j}} \mu^{2 j-n} .
$$

If we substitute $w=1$ and compute the coefficient of $u^{m} v^{\ell}$ in Formula (5), we get the following result:

Corollary 6.2. The number of involutions in $G_{r_{1}, \ldots, r_{k} ; n}$ with exactly $m$ absolute fixed points and $\operatorname{exc}_{A}(\pi)=\ell$ is given by

$$
\left(\frac{n-m}{2}\right) !(r-1)^{\frac{n-m}{2}-\ell}\left(\begin{array}{c}
n \\
\frac{n-m}{2}, m, \frac{n-m}{2}-\ell, \ell
\end{array}\right) \frac{\left(1+2^{\epsilon}\right)^{1-y}}{2^{\frac{n-m}{2}}},
$$

where $y \in\{0,1\}$ and $y \equiv r(\bmod 2)$.

We turn now to the computation of the number of involutions with a fixed number of excedances. We do this by substituting $u=1$ and $v=w^{r}$ in Formula (5).

Corollary 6.3. The number of involutions $\pi \in G_{r_{1}, \ldots, r_{k} ; n}$ with $\operatorname{exc}(\pi)=m$ is:

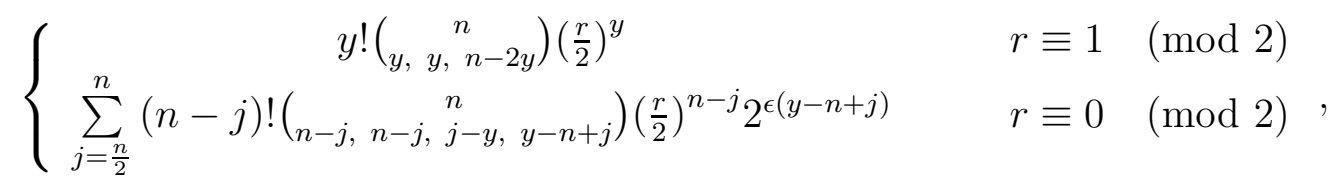

where $y=\frac{m}{r}$. 


\section{Acknowledgments}

The authors wish to thank Robert Schwartz for associating the Dynkin-type diagram to the multi-colored permutation group. We also thank Toufik Mansour for fruitful discussions.

\section{References}

[1] E. Bagno and D. Garber, On the excedance number of colored permutation groups, Semi. Loth. Comb. 53 (2006), Art. B53f, 17 pp. (Electronic, available at

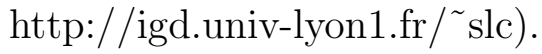

[2] E. Bagno, D. Garber and T. Mansour, Excedance number for involutions in complex reflection groups, Semi. Loth. Comb. 56 (2007), Art. B56d, 11 pp. (Electronic, available at http://igd.univ-lyon1.fr/ ${ }^{\sim}$ slc).

[3] G. Ksavrelof and J. Zeng, Two involutions for signed excedance numbers, Semi. Loth. Comb. 49 (2003), Art. B49e, 8 pp. (Electronic, available at http://igd.univlyon1.fr/ ${ }^{\sim}$ slc).

[4] R. P. Stanley, Enumerative combinatorics, Vol. 1 and 2, Cambridge University Press, 1997. 